\title{
NONLOCAL REGULARIZATION AND SPONTANEOUSLY BROKEN ABELIAN GAUGE THEORIES FOR AN ARBITRARY GAUGE PARAMETER
}

\author{
Anirban Basu
}

October 29, 2018

\author{
Department of Physics,University of Chicago,Chicago IL 60637 \\ Satish D. Joglekar \\ Department of Physics,Indian Institute of Technology,Kanpur 208016 [INDIA]
}

\begin{abstract}
We study the non-local regularization for the case of a spontaneously broken abelian gauge theory in the $\mathrm{R}_{\xi}$-gauge with an arbitrary gauge parameter $\xi$. We consider a simple abelian-Higgs model with chiral couplings as an example. We show that if we apply the nonlocal regularization procedure [to construct a nonlocal theory with FINITE mass parameter ] to the spontaneously broken $\mathrm{R}_{\xi}$-gauge Lagrangian, using the quadratic forms as appearing in this Lagrangian, we find that a physical observable in this model, an analogue of the muon anomalous magnetic moment, evaluated to order $\mathrm{O}\left[\mathrm{g}^{2}\right]$ does indeed show $\xi$ - dependence. We then apply the modified form of nonlocal regularization that was recently advanced and studied for the unbroken non-abelian gauge theories and discuss the resulting WT identities and $\xi$-independence of the S-matrix elements.
\end{abstract}




\section{INTRODUCTION}

The Standard Model [SM] is a renormalizable local quantum field theory [QFT] with a local gauge invariance [1]. Calculations in the SM require the use of a regularization that respects its local gauge invariance. There are various schemes of regularization appropriate for nonabelian gauge theories: the dimensional regularization [DR] , and also a combination of Pauli-Villars and higher derivatives were employed in the early gauge theory literature [2]. However, most attempts to go beyond standard model involve supersymmetry for which a regularization so efficient as the DR cannot be employed.New regularizations have been proposed in the last decade that can be employed in supersymmetric field theories and these are nonlocal regularization $[3,4,5,6,7]$ and differential regularization .For a comparative statement regarding the benefits of the nonlocal regularization vis-a-vis other regularizations, the reader is referred to the Introduction of Ref.[6] and to Ref.5 In this work, we study nonlocal regularization further in some of its aspects.

Nonlocal regularization proposed by Moffat [3] and Evans et al [4] and given an elegant formulation by Kleppe and Woodard $[5,6]$ has been studied extensively since $[7,8]$. Renormalization procedure has been established upto two loop order[5] in scalar theories.In Ref.[6],it has been shown how the nonlocally regularized field theory can be constructed from a local QFT in a systematic fashion. More importantly, it has been established there that the local/global symmetries can be preserved in their nonlocal form and the WT identities of local QFT's derivable from local symmetries such as gauge invariance/BRS symmetry find their natural nonlocal extensions. This has been done for the abelian gauge theories to all orders $[2,6]$ and for nonabelian gauge theories in Feynman gauge [6] upto one loop order [limited,in particular, by the existence of measure beyond one loop]. The nonlocal theories have also found applications in the 
clearer understanding of the renormalization program[14].

As mentioned earlier, we shall study a further aspect of non-local regularization which we shall now elaborate on.In the work of Ref. [6],a procedure for nonlocalization of local Lagrangians was presented. It was further applied to the nonlocalization of the nonabelian gauge theories and to the preservation of its BRS symmetry in a nonlocal form for the Feynman gauge.It was emphasized that the nonlocal theories with a finite $\Lambda$ can themselves be looked upon as physical theories $[3,6,11]$. The significance of this view-point has been elaborated in ref.9 to which the reader is referred to for more details. We shall work in the context of these. In the Ref. [9], which we shall refer to as I henceforth,we studied nonlocal regularization of pure nonabelian unbroken gauge theories with an arbitrary gauge parameter $\xi$. For an arbitrary $\xi$, we established the necessity of a modified treatment for constructing the nonlocal Lagrangian, as compared to that given in [6] for the Feynman gauge. We ,then, gave a way to regularize the nonabelian gauge theory for an arbitrary $\xi$ and established the WT-identities that would imply the $\xi$-independence of the observables for this theory.Now, the SM calculations are calculations in a spontaneously broken gauge theory and that is what is relevant in actual calculations of S-matrix elements. Also, in an unbroken theory, the S-matrix elements may not exist due to infrared divergences and it is harder to test the $\xi$-independence there. In view of these facts ,therefore, we wish to study in this work, the nonlocal regularization in a spontaneously broken model and for an arbitrary $\xi$. Apart from its relevance as mentioned above, we find we have a few other motivations. In a spontaneously broken abelian-Higgs model with chiral couplings to be introduced in Sec.3 [ which in many ways mimics the GWS model, yet is simpler to deal with], we explicitly perform, in this work, the calculation of a simple 1-loop observable: the analogue of the g- 2 for the muon in the model with an arbitrary $\xi$ and find a 
$\xi$-dependent result. An analogous calculation was done earlier by G. Saini and one of us [10] in the context of an actual SM calculation [11] and we had found a $\xi$-dependent result there. The above model of Sec.3 is studied here to explicitly bring out this in a much simpler model with essentially those features that lead to this $\xi$-dependence: viz. spontaneously broken model with chiral couplings. With this motivation in mind,we study the application of the modified way of nonlocal regularization proposed and formulated in unbroken pure Yang-Mills theories to the spontaneously broken theory in I, and study the WT-identities in the theory and the $\xi$-independence of the resulting Green's functions.

We now present the plan of the paper. In Section two, we present the earlier results, viz.:(a) the results in the Ref. 6 on the nonlocalization of a local theory that in particular,preserves the local BRS invariance of the theory in the Feynman gauge,(b) some of the essential results on the nonlocalization procedure, and (c) the conclusions of I regarding the need to modify the general procedure outlined in [6] while dealing with a general $\xi$ and also with calculations in the Feynman gauge in higher loops. In section three, we introduce the abelian- Higgs model with the chiral couplings for an arbitrary $\xi$ and introduce nonlocalization of the $\mathrm{R}_{\xi^{-}}$gauge local Lagrangian following the procedure of the Ref.6. In section four, we evaluate the analogue of the 1-loop contribution to the (g-2) of the muon in this model with a finite mass scale and show that it exhibits $\xi$-independence.In section 5 we study the application of the modified regularization used in I and study the WT-identity.We evaluate $\frac{\partial W}{\partial \xi}$ and simplify the result using the WT-identities. In section 6 , we then discuss the $\xi$-independence of the S-matrix elements.

\section{PRELIMINARIES}

In this section, we shall introduce the notations and review the earlier results on 
the non-local regularization of reference 6 and of I. For brevity, we rely heavily on I, to which the reader is often referred to for any further details.

\section{(A) NON-LOCAL REGULARIZATION:}

We shall, very briefly, review the essential properties of the non-local regularization.We keep the introduction to bare essentials; referring the reader to I for a more detailed introduction.

We write the local action for a field theory ,in terms of a generic field $\phi$, as the sum of the quadratic and the interaction part:

$\mathrm{S}[\phi]=\mathrm{F}[\phi]+\mathrm{I}[\phi]$

and the quadratic piece is expressed as

$\mathrm{F}[\phi]=\int \mathrm{d}^{4} x \phi_{i}(x) \Im_{i j} \phi_{j}(x)$

We define the regularized action in terms of the smeared field $\widehat{\phi}$, defined in terms of the kinetic energy operator $\Im_{i j}$ as,

$\widehat{\phi}=\varepsilon^{-1} \phi$

with $\varepsilon=\exp \left\{\Im / \Lambda^{2}\right\}$. The nonlocally regularized action is constructed by first introducing an auxiliary action $S[\phi, \psi]$.It is given by

$S[\phi, \psi]=\mathrm{F}[\hat{\phi}]-\mathrm{A}[\psi]+\mathrm{I}[\phi+\psi]$

where $\psi$ is called a "shadow field" with an action

$\mathrm{A}[\psi]=\int \mathrm{d}^{4} x \psi_{i} O_{i j}^{-1} \psi_{j}$

with $O$ defined by

$O=\left[\frac{\varepsilon^{2}-1}{\Im}\right]$

The action of the non-local theory is defined as

$\widehat{S}[\phi]=S[\phi, \psi] \|_{\psi=\psi[\phi]}$

where $\psi[\phi]$ if the solution of the classical equation

$0=\frac{\delta S}{\delta \psi}$

The nonlocalized Feynman rules are simple extensions of the local ones. The vertices are unchanged but every leg can connect either to a smeared propagator 
$\frac{i \varepsilon^{2}}{\Im+i \epsilon}=-\mathrm{i} \int_{1}^{\infty} \frac{d \tau}{\Lambda^{2}} \exp \left\{\frac{\Im}{\tau \Lambda^{2}}\right\}$

or to a shadow propagator [a line crossed by a bar]

$\frac{i\left[1-\varepsilon^{2}\right]}{\Im+i \epsilon}=-\mathrm{iO}=-\mathrm{i} \int_{0}^{1} \frac{d \tau}{\Lambda^{2}} \exp \left\{\frac{\Im}{\tau \Lambda^{2}}\right\}$

A set of detailed comments on the Feynman rules and the diagrams that need to be considered are found in I. We only need to remember that in a Feynman diagram, the internal lines can be either shadow or smeared lines with the exception that no diagrams with closed shadow loops are counted.

A number of theorems that finally relate to the construction of the nonlocal symmetry transformations of the nonlocal theory given the local ones for the local theory are established in [6] and are found enumerated in I. We only recall that related to the nonlocal BRS symmetry.

Theorem B.3:[We stick to the theorem numbering of [6] ]. If $\mathrm{S}\{[\phi]\}$ is invariant under $\delta \phi_{i}=\mathrm{T}_{i}[\phi]$, then $\widehat{S}[\phi]$ is invariant under

$\widehat{\delta} \phi_{i}=\varepsilon_{i j}^{2} \mathrm{~T}_{j}[\phi+\psi[\phi]]$

(B) NONLOCAL REGULARIZATION OF A GAUGE THEORY FOR AN ARBITRARY GAUGE PARAMETER

We shall first review briefly the results, in Ref. 6, on nonlocalization of the nonabelian gauge theories in the Feynman gauge. We consider the Feynman gauge local effective action :

$$
\mathrm{S}_{F}=\int \mathrm{d}^{4} x\left[-\frac{1}{2} \partial_{\mu} A_{\nu} \partial^{\mu} A^{\nu}-\partial^{\mu} \bar{\eta} \partial_{\mu} \eta\right]+\mathrm{I}\left[A^{\mu}, \bar{\eta}, \eta\right]
$$

Ref. 6 outlines the results as to how a local action is converted to a non-local action.Briefly put, it is done in the present context by first noting the quadratic operators $\Im_{i j}=\partial^{2} \delta^{4}(\mathrm{x}-\mathrm{y}) \eta_{\mu \nu}$ for the gauge field and $\partial^{2} \delta^{4}(\mathrm{x}-\mathrm{y})$ for the ghost fields and employing these to construct the smeared fields $\widehat{A_{\mu}}=\exp \left\{-\partial^{2} / \Lambda^{2}\right\} \mathrm{A}_{\mu}$ $; \widehat{\eta}=\exp \left\{-\partial^{2} / \Lambda^{2}\right\} \eta ; \hat{\bar{\eta}}=\exp \left\{-\partial^{2} / \Lambda^{2}\right\} \bar{\eta}$. In terms of these, and the corresponding shadow fields $\mathrm{B}_{\mu}{ }^{a}, \psi^{a}, \overline{\psi^{a}}$; we have the non-local action:

$$
\widehat{S_{F}}=\int \mathrm{d}^{4} x\left[-\frac{1}{2} \partial_{\mu} \widehat{A_{\nu}} \partial^{\mu} \widehat{A^{\nu}}-\partial^{\mu} \widehat{\bar{\eta}} \partial_{\mu} \widehat{\eta}-\frac{1}{2} \mathrm{~B}^{a}{ }_{\mu} \mathrm{O}^{-1} \mathrm{~B}^{a \mu}+\overline{\psi^{a}} \mathrm{O}^{-1} \psi^{a}\right]+\mathrm{I}\left[A^{\mu}+B^{\mu}, \bar{\eta}+\right.
$$


$\bar{\psi}, \eta+\psi]$

The local action of (2.11) has a local BRS symmetry.The non-local action ,correspondingly, has a nonlocal BRS symmetry obtained from the result in Sec.2(A). As emphasized in Ref.6, it is found convenient to combine this with a "trivial" i.e. equation of motion symmetry so that the resultant BRS variation of $\bar{\eta}$ is proportional to $\partial . A^{a}$, the field corresponding to the longitudinal degree of freedom. This helps in establishing the WT that establishes the decoupling of the longitudinal degree of freedom. These nonlocal BRS transformations are:

$$
\begin{aligned}
& \widehat{\delta} \mathrm{A}^{a}{ }_{\mu}=\left[\partial_{\mu} \eta^{a}-g \mathrm{f}^{a b c} \varepsilon^{2}\left(\mathrm{~A}_{\mu}+\mathrm{B}_{\mu}\right)^{b}(\eta+\psi)^{c}\right] \delta \zeta \\
& \widehat{\delta} \eta^{a}=-\varepsilon^{2} \frac{g}{2} \mathrm{f}^{a b c} \eta^{b} \eta^{c} \delta \zeta \\
& \widehat{\delta} \bar{\eta}^{a}=-\partial . A^{a} \delta \zeta
\end{aligned}
$$

As emphasized in I, however, when this procedure of nonlocalization as outlined in Sec 2(A) is applied in the straightforward manner, to the case of YangMills theory with an arbitrary gauge parameter $\xi$, it fails to yield WT identities that would yield a $\xi$-independent observables.Moreover,the procedure outlined above in Ref. [6] cannot be employed directly to go beyond 1-loop even for the Feynman gauge,as the gauge parameter $\xi$ gets renormalized and in effect the action in terms of the bare parameters does contain a gauge parameter $\neq 1$. As a consequence, a formulation valid for all $\xi$, must be employed if one is to go beyond 1-loop in this case also.In I, we presented a formulation valid for all $\xi$ and established the relevant WT identities that would imply $\xi$-independence of observables. To illustrate this consider the Yang-Mills effective action for an arbitrary $\xi$ :

$\widehat{S}_{\xi}=\widehat{S}_{F}+\Delta \widehat{S}$

where $\widehat{S}_{F}$ has been given in $(2.12)$ and

$\Delta \widehat{S}=(1-1 / \xi) \int \mathrm{d}^{4} x(\partial . \widehat{A})^{2}$

The smeared field operators depend only on the quadratic form in $\widehat{S}_{F}$. Hence, 
for an arbitrary $\xi$, we have $\widehat{A}_{\mu}=\left(\varepsilon_{F}^{-1}\right)_{\mu \nu} \mathrm{A}^{\nu}=\exp \left\{-\partial^{2} / \Lambda^{2}\right\} \mathrm{A}_{\mu}$ and $\widehat{\eta}=\left(\varepsilon_{\eta}^{-1}\right) \eta=\mathrm{e}$ $\operatorname{xp}\left\{-\partial^{2} / \Lambda^{2}\right\} \eta ; \hat{\bar{\eta}}=\exp \left\{-\partial^{2} / \Lambda^{2}\right\} \bar{\eta}$. We note that $\varepsilon_{F}=\varepsilon_{\eta} \equiv \varepsilon$ here. Further, $O=$ $\left[\frac{\varepsilon^{2}-1}{\Im}\right]$ is the same for $\mathrm{B}$ and for $\psi$ and is independent of $\xi$. The nonlocal BRS symmetry for the case of an arbitrary $\xi$ has been obtained in I.It reads, for an arbitrary $\xi$,

$$
\begin{aligned}
& \widehat{\delta} \mathrm{A}^{a}{ }_{\mu}=\left[\partial_{\mu} \eta^{a}-\mathrm{gf} \mathrm{f}^{a b c} \varepsilon^{2}\left(\mathrm{~A}_{\mu}+\mathrm{B}_{\mu}\right)^{b}(\eta+\psi)^{c}\right] \delta \zeta \\
& \widehat{\delta} \eta^{a}=-\varepsilon^{2} \frac{g}{2} \mathrm{f}^{a b c} \eta^{b} \eta^{c} \delta \zeta \\
& \widehat{\delta} \bar{\eta}^{a}=-\frac{1}{\xi} \partial . A^{a} \delta \zeta
\end{aligned}
$$

In I,we have discussed the WT identities arising from this symmetry and have seen that it leads to a result that would imply the $\xi$-independence of those S-matrix related physical quantities that exist.

\section{NONLOCAL REGULARIZATION OF A SPONTANEOUSLY BROKEN ABELIAN MODEL WITH CHIRAL FERMIONS}

In this work,we shall study an aspect of non-local regularization as related to the spontaneously broken gauge theories. In order to do so,we shall first introduce in this section a simple model - an abelian Higgs Model with Chiral couplings.It is perhaps worthwhile to elaborate and to iterate the context in which we found it essential to study this model.In the work of Ref. [6], a procedure for nonlocalization of nonabelian gauge theories and preservation of the BRS symmetry in a nonlocal form was presented for the Feynman gauge. Subsequently, in I, we studied nonlocal regularization of pure nonabelian unbroken gauge theories with an arbitrary gauge parameter $\xi$. For an arbitrary $\xi$, we established the necessity of a modified treatment for constructing the nonlocal Lagrangian as compared to that given in [6] for the Feynman gauge. We ,then, gave a way of regularize the nonabelian gauge theory for an arbitrary $\xi$ and established the WT-identities that would imply the $\xi$-independence of the observables for 
this theory. In view of the fact that the calculation of an observable in the SM generally involves a spontaneously broken gauge theory, the extension of an analogous treatment to the case of spontaneously broken case is of value.In this work, therefore, we wish to study, for an arbitrary $\xi$, the nonlocal regularization in a spontaneously broken model. Apart from its relevance as mentioned above, we find we have a few other motivations. In section IV, we shall perform the calculation of a simple 1-loop observable: the analogue of the g-2 for the muon in the model with arbitrary $\xi$ explicitly and find a $\xi$-dependent result. An analogous calculation was done earlier by G. Saini and one of us [10 ] in the context of an actual SM calculation[11] and we had found a $\xi$-dependent result.The above model is studied here to explicitly bring out this in a simpler model with essentially those features that lead to this: viz. spontaneously broken model with chiral couplings. With this motivation in mind, we study the application of the modified way of nonlocal regularization proposed and formulated in unbroken pure Yang-Mills theories to the spontaneously broken theory in I and study the WT-identities in the theory and the $\xi$-independence of the resulting Green's functions. Therefore, in this section,we shall introduce an abelian Higgs model with gauge fields coupling to vector and the axial vector currents. As mentioned earlier, this simpler model incorporates in it several essential features of the standard model such as coupling of gauge fields to chiral fermions and a spontaneously broken nature of the model. Both these features are essential in the example that we shall discuss in the next section.

Consider the Lagrangian for the model with a local U(1)XU(1) symmetry ,having a complex scalar $\varphi$, a fermion $\psi$ and two $\mathrm{U}(1)$ gauge fields $\mathrm{A}$ and $\mathrm{B}$, coupling respectively to vector and the axial vector currents and the quantum 
numbers for the fields as indicated below:"

\begin{tabular}{|c|c|c|}
\hline & $\mathrm{U}_{V}(1)$ & $\mathrm{U}_{A}(1)$ \\
\hline \hline$\varphi$ & 0 & 2 \\
\hline$\psi_{L}$ & 1 & 1 \\
\hline$\psi_{R}$ & 1 & -1 \\
\hline
\end{tabular}

Table 1:Quantum Numbers

$$
\begin{aligned}
& \mathrm{L}_{i n v}=\left(\mathrm{D}_{\mu} \varphi\right)^{\dagger}\left(\mathrm{D}^{\mu} \varphi\right)-\mu^{2} \varphi^{\dagger} \varphi-\lambda\left(\varphi^{\dagger} \varphi\right)^{2}-1 / 4 \mathrm{~F}_{\mu \nu} \mathrm{F}^{\mu \nu}-1 / 4 \mathrm{G}_{\mu \nu} \mathrm{G}^{\mu \nu}+\bar{\psi}_{L}(i \not \partial+\mathrm{gB}- \\
& \mathrm{eAA}) \psi_{L}+\bar{\psi}_{R}(i \not \partial \text {-gB-eA }) \psi_{R}+\mathrm{f}\left(\bar{\psi}_{L} \psi_{R} \varphi+\overline{\psi_{R}} \psi_{L} \varphi^{*}\right)
\end{aligned}
$$

where,

$$
\begin{aligned}
& \varphi=\frac{\varphi_{1}+i \varphi_{2}}{\sqrt{2}} ; \varphi_{1}, \text { and } \varphi_{2} \text { real. } \\
& \mathrm{D}_{\mu}=\partial_{\mu}-2 \mathrm{igB} B_{\mu} \\
& \mathrm{F}_{\mu v}=\partial_{\mu} \mathrm{A}_{\nu}-\partial_{\nu} A_{\mu} \\
& \mathrm{G}_{\mu v}=\partial_{\mu} \mathrm{B}_{\nu}-\partial_{\nu} B_{\mu} ;
\end{aligned}
$$

the $(\text { mass })^{2}$ parameter $=\mu^{2}<0$ and $\varphi$ develops a vacuum expectation value given by:

$$
\left\langle\varphi^{\dagger} \varphi\right\rangle=-\mu^{2} / 2 \lambda \equiv \frac{v^{2}}{2}>0 .
$$

Further, the gauge-fixing Lagrangian in the $R_{\xi}$-gauges is given by[]

$$
\mathrm{L}_{g f}=-1 / 2(\partial . A)^{2}-\frac{1}{2 \xi}\left(\partial . \mathrm{B}+\xi \mathrm{M} \varphi_{2}\right)^{2}, \mathrm{M} \equiv 2 \mathrm{gv}
$$

Thus, we can now re-express the net Lagrangian in terms of the shifted fields: $\varphi_{1}^{\prime}=\varphi_{1}-\mathrm{v}$, and $\varphi_{2}^{\prime}=\varphi_{2}$ as $\mathrm{L}=\mathrm{L}_{0}+\mathrm{L}_{I}$, with:

$$
\begin{aligned}
& \mathrm{L}_{0}=\frac{1}{2}\left[\left(\partial_{\mu} \varphi_{1}^{\prime}\right)^{2}+2 \mu^{2} \varphi_{1}{ }^{2}\right]+\frac{1}{2}\left[\left(\partial_{\mu} \varphi_{2}^{\prime}\right)^{2}-\mathrm{M}^{2} \xi \varphi_{2}{ }^{\prime 2}\right]-\frac{1}{4}\left(\partial_{\mu} \mathrm{B}_{\nu}-\partial_{\mu} \mathrm{B}_{\nu}\right)\left(\partial^{\mu} \mathrm{B}_{\nu}-\partial^{\nu} \mathrm{B}^{\mu}\right) \\
& +\frac{1}{2} \mathrm{M}^{2} \mathrm{~B}_{\mu} \mathrm{B}^{\mu}-\frac{1}{2 \xi}(\partial . \mathrm{B})^{2}-\frac{1}{2} \partial_{\mu} \mathrm{A}_{\nu} \partial^{\mu} \mathrm{A} \nu+\bar{\psi}(i \not \partial-\mathrm{m}) \psi \\
& \mathrm{L}_{I}=\bar{\psi}_{L}(\mathrm{gB}-\mathrm{e} \mathrm{A}) \psi_{L}+\bar{\psi}_{R}(-\mathrm{gB}-\mathrm{eA}) \psi_{R^{-}} \frac{m}{v} \bar{\psi}_{L} \psi_{R}\left(\varphi_{1}^{\prime}+\mathrm{i} \varphi_{2}^{\prime}\right)-\frac{m}{v} \overline{\psi_{R}} \psi_{L}\left(\varphi_{1}^{\prime}-\mathrm{i} \varphi_{2}^{\prime}\right) \\
& \quad+2 \mathrm{gB} \mathrm{B}^{\mu}\left(\varphi_{2}^{\prime} \partial_{\mu} \varphi_{1}^{\prime}-\varphi_{1}^{\prime} \partial_{\mu} \varphi_{2}^{\prime}\right)+2 \mathrm{~g}^{2} \mathrm{~B}_{\mu} \mathrm{B}^{\mu}\left(\varphi_{1}^{\prime 2}+\varphi_{2}^{\prime 2}\right)-\frac{\lambda}{4}\left(\varphi_{1}^{\prime 2}+\varphi_{2}^{\prime 2}\right)^{2}+4 \mathrm{~g}^{2} \mathrm{vB}_{\mu} \mathrm{B}^{\mu} \varphi_{1}^{\prime}- \\
& \lambda \mathrm{v} \varphi_{1}^{\prime}\left(\varphi_{1}^{\prime 2}+\varphi_{2}^{\prime 2}\right) ;
\end{aligned}
$$

\footnotetext{
${ }^{1}$ The model below has the chiral anomaly.It can be removed by adding another fermion $\Psi$ with electric charge \pm 1 and opposite axial charge. Introduction of $\Psi$ does not alter the one-loop calculation of (g-2) for $\psi$ presented in the next section. Hence, to this order we ignore the question of the anomaly .

${ }^{2}$ We keep the gauge parameter associated the unbroken $\mathrm{U}(1)$ field equal to 1.
} 
here $\mathrm{m}=-\mathrm{fv} / \sqrt{2}$ stands for the fermion mass.

We now assumes that the procedure for nonlocalizing the above local Lagrangian is as elaborated in Sec. 2(A).To this end, we recall the quadratic structures in $\mathrm{L}_{0}$ and introduce the shadow fields for each of the fields in $\mathrm{L}$ as indicated in the following table:

\begin{tabular}{|c|c|c|c|c|c|}
\hline fields & $\varphi_{1}^{\prime}$ & $\varphi_{2}^{\prime}$ & $\psi$ & $\mathrm{A}$ & $\mathrm{B}$ \\
\hline \hline shadow fields & $\rho_{1}$ & $\rho_{2}$ & $\varsigma$ & $\mathrm{H}$ & $\mathrm{D}$ \\
\hline
\end{tabular}

Table 2

\begin{tabular}{|c|c|}
\hline field & propagator \\
\hline \hline$\varphi_{1}^{\prime}$ & $-\mathrm{i} \int_{1}^{\infty} \frac{d \tau}{\Lambda^{2}} \exp \left\{\frac{\tau}{\Lambda^{2}}\left(\mathrm{k}^{2}+2 \mu^{2}\right)\right\}$ \\
\hline$\varphi_{2}^{\prime}$ & $-\mathrm{i} \int_{1}^{\infty} \frac{d \tau}{\Lambda^{2}} \exp \left\{\frac{\tau}{\Lambda^{2}}\left(\mathrm{k}^{2}-\mathrm{M}^{2} \xi\right)\right\}$ \\
\hline$\psi$ & $-\mathrm{i} \int_{1}^{\infty} \frac{d \tau}{\Lambda^{2}} \exp \left\{\frac{\tau}{\Lambda^{2}}\left(\mathrm{k}^{2}-\mathrm{m}^{2}\right)\right\}(\mathrm{k}+\mathrm{m})$ \\
\hline $\mathrm{A}$ & $\mathrm{i} \int_{1}^{\infty} \frac{d \tau}{\Lambda^{2}} \exp \left\{\frac{\tau}{\Lambda^{2}} \mathrm{k}^{2}\right\} \eta_{\mu \nu}$ \\
\hline $\mathrm{B}$ & $\mathrm{i} \int_{1}^{\infty} \frac{d \tau}{\Lambda^{2}} \exp \left\{\frac{\tau}{\Lambda^{2}}\left(\mathrm{k}^{2}-M^{2}\right)\right\}\left[\eta_{\mu \nu}-\frac{k_{\mu} k_{\nu}}{k^{2}}\left\{1-\exp \left(-\frac{\tau}{\Lambda^{2}} \mathrm{k}^{2}\left(1-\frac{1}{\xi}\right)\right)\right\}\right] ;$ \\
\hline
\end{tabular}

Table 3

\begin{tabular}{|c|c|}
\hline shadow field & propagator \\
\hline \hline$\rho_{1}$ & $-\mathrm{i} \int^{1} 0 \frac{d \tau}{\Lambda^{2}} \exp \left\{\frac{\tau}{\Lambda^{2}}\left(\mathrm{k}^{2}+2 \mu^{2}\right)\right\} ;$ \\
\hline$\rho_{2}$ & $-\mathrm{i} \int_{0}^{1} \frac{d \tau}{\Lambda^{2}} \exp \left\{\frac{\tau}{\Lambda^{2}}\left(\mathrm{k}^{2}-\mathrm{M}^{2} \xi\right)\right\} ;$ \\
\hline$\varsigma$ & $-\mathrm{i} \int_{0}^{1} \frac{d \tau}{\Lambda^{2}} \exp \left\{\frac{\tau}{\Lambda^{2}}\left(\mathrm{k}^{2}-\mathrm{m}^{2}\right)\right\}(\mathrm{k}+\mathrm{m})$ \\
\hline $\mathrm{H}$ & $\mathrm{i} \int_{0}^{1} \frac{d \tau}{\Lambda^{2}} \exp \left\{\frac{\tau}{\Lambda^{2}} \mathrm{k}^{2}\right\} \eta_{\mu \nu} ;$ \\
\hline $\mathrm{D}$ & $\mathrm{i} \int^{1} \frac{d \tau}{\Lambda^{2}} \exp \left\{\frac{\tau}{\Lambda^{2}}\left(\mathrm{k}^{2}-M^{2}\right)\right\}\left[\eta_{\mu \nu}-\frac{k_{\mu} k_{\nu}}{k^{2}}\left\{1-\exp \left(-\frac{\tau}{\Lambda^{2}} \mathrm{k}^{2}\left(1-\frac{1}{\xi}\right)\right)\right\}\right]$ \\
\hline
\end{tabular}

Table 4

\section{A CALCULATION TO TEST $\xi$-INDEPENDENCE}

In this section,we shall present a calculation to test the $\xi$-dependence of a phys-

\footnotetext{
${ }^{3}$ In the case of spontaneously broken gauge theories, there are potentially two ways of nonlocalising. The one is as shown here in this work. The other is to start from the model in an unbroken form, nonlocalize the model and then break symmetry.In the latter case,we cannot , of course, add the gauge fixing in the $\mathrm{R}_{\xi}$-form in the unbroken action. However, the entire procedure in the latter case turns out to be very cumbersome.
} 
ical quantity. The quantity we consider is the analogue, in the context of the present model,of the (g-2) of a muon in the standard model.The calculation proceeds very much along the lines of the reference [11], where the $(\mathrm{g}-2)$ of the muon was calculated in the non-local Standard model.The essential points to note in this 1-loop calculation are :

(a) The quantity we are calculating is finite both in the local and the nonlocal theories; hence so is the difference. This difference is of order $\mathrm{O}\left[\frac{m^{2}}{\Lambda^{2}}\right]$ as compared to the local result for $(\mathrm{g}-2)$.

(b) The difference between the local and the nonlocal results is given by the various 1-loop diagrams having a shadow loop; and the total of these as seen above are finite in value;

(c) As a result, the conclusion of the calculation we are to perform below, is independent of a renormalization procedure;

(d) The local contribution is known to be $\xi$-independent; so that the $\xi$ independence, or the lack of it, is determined by the finite 1-loop shadow diagrams.

We enumerate these diagrams involved .These are shown in Fig.1 and correspond to the exchanges of $\mathrm{H}, \mathrm{D}, \rho_{1}, \rho_{2}$.Noting that vertices are $\xi$-independent, a glance at the Table 4 in Section 3 will show that the $\xi$-dependence of the result can possibly come only from the $\mathrm{D}$ and the $\rho_{2}$-exchange. These are the diagrams of Fig.1(b) and 1(d).

In evaluating the $\xi$-dependence to the leading order, the following observation about a loop integral comes handy: Consider an integral of the form

$\frac{1}{\Lambda^{4}} \int \frac{d^{4} l}{(2 \pi)^{4}} \exp \left\{\alpha(\xi) l^{2} / \Lambda^{2}+\beta(\xi) \mathrm{m}^{2} / \Lambda^{2}\right\}$

We can evaluate the above integral by expanding $\exp \left\{\beta(\xi) \mathrm{m}^{2} / \Lambda^{2}\right\}$ in powers of $\beta(\xi) \mathrm{m}^{2} / \Lambda^{2}$ since each term is a finite integral.Then, we have

$\frac{1}{\Lambda^{4}} \int \frac{d^{4} l}{(2 \pi)^{4}} \exp \left\{\alpha(\xi) l^{2} / \Lambda^{2}+\beta(\xi) \mathrm{m}^{2} / \Lambda^{2}\right\}=\frac{A}{\alpha(\xi)^{2}}+$ terms of higher order in 
$1 / \Lambda^{2}$

Thus, as far as the leading terms arising from (4.1) are concerned, the contribution to the $\xi$-dependence from $\beta(\xi) \mathrm{m}^{2} / \Lambda^{2}$ can be ignored; and to the leading order, the $\xi$-dependence of $(4.1)$ is determined by $\alpha(\xi)$.

With this in mind, we turn our attention to the diagrams $1(\mathrm{~b})$ and $1(\mathrm{~d})$.We recall the propagator of $\rho_{2}$ in $1(\mathrm{~d})$ from the Table 4 . We shall first show that the diagram of Fig.1(d) does not have $\xi$-dependent term on the leading order [ $\left.\mathrm{O}\left(\mathrm{m}^{2} / \Lambda^{2}\right)\right]$.To see this, we write out the expression for the diagram using the propagators from Table 4:

$$
\begin{aligned}
& \mathrm{i} \Delta \Gamma_{\alpha}^{(1)}=\int \frac{d^{4} l}{(2 \pi)^{4}}\left[-\mathrm{i} \int_{0}^{1} \frac{d \tau_{3}}{\Lambda^{2}} \exp \left\{\frac{\tau_{3}}{\Lambda^{2}}\left((\mathrm{p}-\mathrm{l})^{2}-\mathrm{M}^{2} \xi\right)\right\}\right] \overline{u(q)} \frac{m}{v} \gamma_{5} \\
& \bullet\left[-\mathrm{i} \int_{0}^{1} \int \frac{d \tau_{1}}{\Lambda^{2}} \exp \left\{\frac{\tau_{1}}{\Lambda^{2}}\left((l-p+q)^{2}-\mathrm{m}^{2}\right)\right\}(l-\not b+\not l+\mathrm{m})\right]\left(-\mathrm{i} e \gamma_{\alpha}\right)\left[-\mathrm{i} \int_{0}^{1} \frac{d \tau_{2}}{\Lambda^{2}} \exp \left\{\frac { \tau _ { 2 } } { \Lambda ^ { 2 } } \left(l^{2}-\right.\right.\right. \\
& \left.\left.\left.\mathrm{m}^{2}\right)\right\}(l+\mathrm{m})\right] \frac{m}{v} \gamma_{5} \mathrm{u}(\mathrm{p})
\end{aligned}
$$

We now let $l->l+\frac{\tau_{1+} \tau_{3}}{\tau} p-\frac{\tau_{1}}{\tau} q$; with $\tau=\tau_{1+} \tau_{2+} \tau_{3}$. This gives

$\mathrm{i} \Delta \Gamma_{\alpha}^{(1)}(\mathrm{p}, \mathrm{q})=\mathrm{e}\left[\frac{m}{v}\right]^{2} \int \frac{d^{4} l}{(2 \pi)^{4}} \int_{0}^{1} \frac{d \tau_{1}}{\Lambda^{2}} \int_{0}^{1} \frac{d \tau_{2}}{\Lambda^{2}} \int_{0}^{1} \frac{d \tau_{3}}{\Lambda^{2}} \overline{u(q)} \gamma_{5}\left(l-\frac{\tau_{2}}{\tau} \not p+\frac{\tau_{3+} \tau_{2}}{\tau} \not 1+\mathrm{m}\right) \gamma_{\alpha}\left(\not l+\frac{\tau_{1+} \tau_{2}}{\tau} \not p-\right.$ $\frac{\tau_{1}}{\tau} \phi(\mathrm{m}) \gamma_{5} \mathrm{u}(\mathrm{p}) \exp \left\{\frac{\tau}{\Lambda^{2}} \mathrm{l}^{2}-2 \frac{\tau_{1} \tau_{2}}{\tau \Lambda^{2}} p \cdot q-\frac{1}{\Lambda^{2}}\left[\tau_{3} \mathrm{M}^{2} \xi+\frac{\tau_{1^{2}{ }^{2}} \tau_{2}^{2}}{\tau} m^{2}\right)\right\}$

We now recall the remark made below (4.2). The above expression, as result of this remark, does not have a $\xi$-dependent contribution to (g-2) to the leading order $\left[\mathrm{O}\left(\mathrm{m}^{2} / \Lambda^{2}\right)\right]$.

We now turn our attention to the diagram 1(b).The expression for this shadow diagram is

$$
\Delta \Gamma_{\alpha}^{(2)}=\int \frac{d^{4} l}{(2 \pi)^{4}} \overline{u(q)}\left(-\operatorname{ig} \gamma^{\mu} \gamma_{5}\right)\left[\operatorname { i } \int _ { 0 } ^ { 1 } \frac { d \tau _ { 1 } } { \Lambda ^ { 2 } } \operatorname { e x p } \{ \frac { \tau _ { 1 } } { \Lambda ^ { 2 } } ( l ^ { 2 } - M ^ { 2 } ) \} \left[\eta _ { \mu \nu ^ { - } } \frac { l _ { \mu } l _ { \nu } } { l ^ { 2 } } \left\{1-\exp \left(-\frac{\tau_{1}}{\Lambda^{2}} l^{2}(1-\right.\right.\right.\right.
$$
$\left.\left.\left.\left.\frac{1}{\xi}\right)\right)\right\}\right]$

$$
\text { - }\left[-\mathrm{i} \int_{0}^{1} \frac{d \tau_{2}}{\Lambda^{2}} \exp \left\{\frac{\tau_{2}}{\Lambda^{2}}\left((l-q)^{2}-\mathrm{m}^{2}\right)\right\}(-\not+\not q+\mathrm{m})\right]\left(-\mathrm{i} e \gamma_{\alpha}\right)\left[-\mathrm{i} \int_{0}^{1} \frac{d \tau_{3}}{\Lambda^{2}} \exp \left\{\frac{\tau_{3}}{\Lambda^{2}}\left((\mathrm{p}-l)^{2}-\mathrm{m}^{2}\right)\right\}\right.
$$
$(\not p-l+\mathrm{m})]\left(-\operatorname{ig} \gamma^{\nu} \gamma_{5}\right) \mathrm{u}(\mathrm{p})$

For reasons mentioned in paragraph below eq.(4.2), it is easily seen that the $\eta_{\mu \nu}$ term in the D-propagator cannot contribute to the $\xi$-dependence of the ( $\mathrm{g}$ 2 ) in the leading order. Thus we need to consider the contribution of the $l_{\mu} l_{\nu} / l^{2}$ 
term only. These terms contribute essentially due to the chiral nature of vertices and give a contribution proportional to $\mathrm{m}^{2}$. The result for terms that contribute to $(\mathrm{g}-2)$ reads:

$$
\Delta \Gamma_{\mu}^{(2)}=\frac{e}{2 m} \frac{g^{2}}{2 \pi^{2}} \frac{m^{2}}{\Lambda^{2}} \overline{u(q)} \sigma_{\mu \nu} \mathrm{u}(\mathrm{p})(\mathrm{p}-\mathrm{q})^{\nu} \int_{0}^{1} \mathrm{~d} \tau_{1} \int_{0}^{1} d \tau_{2} \int_{0}^{1} \mathrm{~d} \tau_{3}
$$

- $\left[\xi\right.$-independent terms from the $\eta_{\mu \nu^{-}}$term $\left.+\frac{\tau_{2}}{3\left[\tau+\tau_{1}(1-1 / \xi)\right]^{3}}-\frac{\tau_{3} \tau_{2}}{4\left[\tau+\tau_{1}(1-1 / \xi)\right]^{4}}\right]+[$ $\left.\mathrm{O}\left(\mathrm{m}^{2} / \Lambda^{2}\right)^{2}\right]$.

A direct evaluation of the above integral shows that it is $\xi$-dependent.

We thus note the presence of $\xi$-dependent terms of $\mathrm{O}\left[\frac{m^{2}}{\Lambda^{2}}\right]$ which are the only $\xi$-dependent terms contributing to (g-2).

We summarize the result of this section. We considered an abelian Higgs model with axial and vector couplings. We then wrote down the spontaneously broken $R_{\xi}$-gauge local action and nonlocalized the action using the recipe of the reference 6 for an arbitrary $\xi$.We then considered a physically measurable quantity viz. (g-2) of the fermion to check the $\xi$-dependence of the result.We note that the calculation of this quantity in 1-loop does not depend on renormalizations that need to be carried out.We noted that the nonlocal contribution was finite and given by the negative of the relevant shadow diagrams. We isolated the diagrams that could be $\xi$-dependent.We then showed that the $\rho_{2}$-exchange diagram does not lead to a $\xi$-dependence in the leading order contribution to (g-2). We evaluated the contribution to (g-2) from the diagram with the Dexchange and it turned out to have $\xi$-dependence in order $\mathrm{O}\left[\frac{m^{2}}{\Lambda^{2}}\right]$.

Keeping in mind that the mass here has arisen due to spontaneous symmetry breakdown, we see that the above demonstration of gauge-dependence has been dependent on the two features of a spontaneously broken model and of having the axial couplings: both of which are present in the standard model. In fact a calculation along the lines of reference 11, gives a $\xi$-dependent result for ( $\mathrm{g}$ 2 ) of the muon in the standard model [10] if the above procedure is used for 
nonlocalization there.

\section{NONLOCAL REGULARIZATION FOR AN ARBITRARY $\xi$ AND THE WT-IDENTITIES}

As shown in the previous section, the general procedure of nonlocalization, when applied to the spontaneously broken local action in $\mathrm{R}_{\xi}$-gauges for the model considered there, leads to a $\xi$-dependent physical observable. In I, we had formulated an alternate procedure for nonlocalization of the nonabelian gauge theories and established a WT-identity that would imply the $\xi$-independence of the physical observables. We wish to carry out an analogous exercise here for the model presented in section 3. [We note that since this is a spontaneously broken model,a larger class of S-matrix elements themselves (rather than crosssections with an energy cut-off) exist.]We shall write down the WT identities for the model and obtain $\left.\frac{\partial W}{\partial \xi}\right|_{\xi=1}$. Then, in the next section,we shall discuss the gauge-independence of the S-matrix using these results.

$$
\begin{aligned}
& \mathrm{L}_{B R S}=\mathrm{L}_{i n v}+\mathrm{L}_{g f}+\mathrm{L}_{g h} \\
& =\left(\mathrm{D}_{\mu} \varphi\right)^{\dagger}\left(\mathrm{D}^{\mu} \varphi\right)-\mu^{2} \varphi^{\dagger} \varphi-\lambda\left(\varphi^{\dagger} \varphi\right)^{2}-\frac{1}{2} \partial_{\mu} \mathrm{A}_{\nu} \partial^{\mu} \mathrm{A}_{\nu}-1 / 4 \mathrm{G}_{\mu \nu} \mathrm{G}^{\mu \nu}+\bar{\psi}_{L}(i \not \partial+\mathrm{gB}-\mathrm{e} A) \psi_{L} \\
& +\bar{\psi}_{R}(i \not \partial-\mathrm{gB}-\mathrm{e} A) \psi_{R}+\frac{\sqrt{2} m}{v}\left(\bar{\psi}_{L} \psi_{R} \varphi+\overline{\psi_{R}} \psi_{L} \varphi^{*}\right)-\frac{1}{2}\left(\partial . \mathrm{B}+\xi \mathrm{M} \varphi_{2}{ }^{2}\right)^{2}+\partial^{\mu} \bar{\eta} \partial_{\mu} \eta-
\end{aligned}
$$

$2 \mathrm{Mg} \varphi_{1} \bar{\eta} \eta$

has the following BRS symmetry [arising from the $\mathrm{U}_{A}(1)$ local symmetry] in the Feynman gauge $\xi=1$ :

$$
\begin{aligned}
& \delta \varphi_{1}=2 \eta \varphi_{2} \delta \zeta ; \delta \eta=0 ; \delta B_{\mu}=-\frac{1}{g} \partial_{\mu} \eta \delta \zeta \\
& \delta \varphi_{2}=-2 \eta \varphi_{1} \delta \zeta ; \delta \bar{\eta}=-\frac{1}{g}\left(\partial . \mathrm{B}+\mathrm{M} \varphi_{2}\right) \delta \zeta ; \delta A_{\mu}=0 \\
& \delta \psi=-\mathrm{i} \eta \gamma_{5} \psi \delta \zeta
\end{aligned}
$$

The nonlocal Feynman gauge action is obtained by using the quadratic forms in (5.1) and following the procedure as in Sec 2. It reads,

$$
\widehat{S}_{F}=\int \mathrm{d}^{4} x\left\{\frac{1}{2} \widehat{\varphi_{1}^{\prime}}\left(-\partial^{2}+2 \mu^{2}\right) \widehat{\varphi_{1}^{\prime}}+\frac{1}{2} \widehat{\varphi_{2}^{\prime}}\left(-\partial^{2}-M^{2}\right) \widehat{\varphi_{2}^{\prime}}+\frac{1}{2} \widehat{B}_{\mu}\left(\partial^{2}+M^{2}\right) \widehat{B}^{\mu}+\frac{1}{2} \widehat{A}_{\mu}\right.
$$




$$
\begin{aligned}
& \partial^{2} \widehat{A}^{\mu} \\
& \left.+\widehat{\bar{\psi}} \mathrm{i} \partial \hat{\psi}-\mathrm{m} \widehat{\bar{\psi}} \hat{\psi}-\widehat{\bar{\eta}} \partial^{2} \widehat{\eta}-2 \mathrm{Mgv} \hat{\bar{\eta}} \widehat{\eta}\right\}-\int \mathrm{d}^{4} x\left\{\frac{1}{2} \rho_{1} \mathrm{O}^{-1} \varphi_{1}^{\prime} \rho_{1}+\frac{1}{2} \rho_{2} \mathrm{O}^{-1} \varphi_{2}^{\prime} \rho_{2}\right. \\
& \left.+\frac{1}{2} \mathrm{D}^{\mu} \mathrm{O}^{-1}{ }_{B} \mathrm{D}_{\mu}+\frac{1}{2} \mathrm{H}^{\mu} \mathrm{O}^{-1}{ }_{A} \mathrm{H}_{\mu}+\bar{\zeta} \mathrm{O}^{-1}{ }_{\psi} \zeta-\bar{c} \mathrm{O}^{-1}{ }_{\eta} \mathrm{c}\right\}+\mathrm{I} \text {, } \\
& \mathrm{I}=\int \mathrm{d}^{4} x\left\{-2 \mathrm{Mg}\left(\varphi_{1}{ }^{\prime}+\rho_{1}\right)(\bar{\eta}+\bar{c})(\eta+c)+2 \mathrm{~g}(\mathrm{~B}+\mathrm{D})^{\mu}\left[\left(\varphi_{2}{ }^{\prime}+\rho_{2}\right) \partial_{\mu}\left(\varphi_{1}{ }^{\prime}+\rho_{1}\right)-\right.\right. \\
& \left.\left(\varphi_{1}{ }^{\prime}+\rho_{1}\right) \partial_{\mu}\left(\varphi_{2}{ }^{\prime}+\rho_{2}\right)\right] \\
& +2 \mathrm{~g}^{2}(\mathrm{~B}+\mathrm{D})^{2}\left[\left(\varphi_{2}{ }^{\prime}+\rho_{2}\right)^{2}+\left(\varphi_{1}{ }^{\prime}+\rho_{1}\right)^{2}\right]-\frac{\lambda}{4}\left[\left(\varphi_{2}{ }^{\prime}+\rho_{2}\right)^{2}+\left(\varphi_{1}{ }^{\prime}+\rho_{1}\right)^{2}\right]^{2} \\
& +4 \mathrm{~g}^{2} \mathrm{v}(\mathrm{B}+\mathrm{D})^{2}\left(\varphi_{1}{ }^{\prime}+\rho_{1}\right)-\lambda \mathrm{v}\left(\varphi_{1}{ }^{\prime}+\rho_{1}\right)\left[\left(\varphi_{2}{ }^{\prime}+\rho_{2}\right)^{2}+\left(\varphi_{1}{ }^{\prime}+\rho_{1}\right)^{2}\right] \\
& +\left(\bar{\psi}_{L}+\bar{\zeta}_{L}\right)[\mathrm{g}(\not B+\not D)-\mathrm{e}(\not A+\not A)]\left(\psi_{L}+\zeta_{L}\right)+\left(\bar{\psi}_{R}+\overline{\zeta_{R}}\right)[-\mathrm{g}(\not B+\not D)-\mathrm{e}(\not A+H)]\left(\psi_{R}+\zeta_{R}\right) \\
& -\frac{m}{v}\left(\bar{\psi}_{L}+\bar{\zeta}_{L}\right)\left[\varphi_{1}{ }^{\prime}+\rho_{1}+i \varphi_{2}{ }^{\prime}+\mathrm{i} \rho_{2}\right]\left(\psi_{R}+\zeta_{R}\right) \\
& -\frac{m}{v}\left(\bar{\psi}_{R}+\overline{\zeta_{R}}\right)\left[\varphi_{1}{ }^{\prime}+\rho_{1}-i \varphi_{2}{ }^{\prime}-\mathrm{i} \rho_{2}\right]\left(\psi_{L}+\zeta_{L}\right)
\end{aligned}
$$

The nonlocalized symmetries of the nonlocal action in the Feynman gauge then are

$$
\begin{aligned}
& \widehat{\delta} \varphi_{1}^{\prime}=2 \varepsilon_{\varphi_{1}^{\prime}}^{2}\left(\varphi_{2}{ }^{\prime}+\rho_{2}\right)(\eta+c) \delta \zeta ; \\
& \widehat{\delta} \varphi_{2}^{\prime}=-2 \varepsilon_{\varphi_{2}^{\prime}}^{2}\left(\varphi_{1}{ }^{\prime}+\rho_{1}+\mathrm{v}\right)(\eta+c) \delta \zeta ; \\
& \widehat{\delta} \eta=0 ; \\
& \widehat{\delta} \bar{\eta}=-\frac{1}{g} \varepsilon_{\eta}^{2}\left[\partial .(\mathrm{B}+\mathrm{D})+\mathrm{M}\left(\varphi_{2}{ }^{\prime}+\rho_{2}\right)\right] \delta \zeta ; \\
& \widehat{\delta} B_{\mu}=-\frac{1}{g} \varepsilon_{B \mu \nu}^{2} \partial^{\nu}(\eta+c) \delta \zeta ; \\
& \widehat{\delta} A_{\mu}=0,
\end{aligned}
$$

In addition, we shall find it convenient to employ the trivial symmetry as in Ref. [6] .When it is added to those in (5.5a), the net symmetry transformations read:

$$
\begin{aligned}
& \widehat{\delta} \varphi_{1}^{\prime}=2 \varepsilon_{\varphi_{1}^{\prime}}^{2}\left(\varphi_{2}{ }^{\prime}+\rho_{2}\right)(\eta+c) \delta \zeta ; \\
& \widehat{\delta} \varphi_{2}^{\prime}=\left\{-2 \varepsilon_{\varphi_{2}^{\prime}}^{2}\left(\varphi_{1}{ }^{\prime}+\rho_{1}\right)(\eta+c)-2 v \eta\right\} \delta \zeta ; \\
& \widehat{\delta} \eta=0 ; \\
& \widehat{\delta} \bar{\eta}=-\frac{1}{g}\left[\partial \cdot \mathrm{B}+\mathrm{M} \varphi_{2}{ }^{\prime}\right] \delta \zeta ; \\
& \widehat{\delta} B_{\mu}=-\frac{1}{g} \partial_{\mu} \eta \delta \zeta ;
\end{aligned}
$$


$\widehat{\delta} A_{\mu}=0$,

We note the essential similarity with the nonlocal Y-M theory:The linear parts of the net transformation are same as the local case, while the quadratic parts of the net transformation are unmodified by the trivial transformation. We note that the measure for the nonlocal theory will be determined with respect to these non-local transformations of (5.5b).

In an arbitrary gauge $\xi$, we follow the procedure of I [see also ref.7], and smear the fields with the quadratic forms of the Feynman gauge action of ( 5.1). The nonlocalized action then reads

$$
\begin{aligned}
& \widehat{S}_{\xi}=\widehat{S}_{F}+\Delta \widehat{S} \\
& \Delta \widehat{S}=\int \mathrm{d}^{4} x\left\{-\frac{1}{2 \xi}\left(\partial . \widehat{B}+\xi \mathrm{M} \widehat{\varphi_{2}^{\prime}}\right)^{2}+\frac{1}{2}\left(\partial . \widehat{B}+\mathrm{M} \widehat{\varphi_{2}^{\prime}}\right)^{2}+2(1-\xi) \operatorname{gMv} \int \mathrm{d}^{4} x \hat{\bar{\eta}} \widehat{\eta}\right. \\
& \left.+2(1-\xi) \operatorname{gM} \int \mathrm{d}^{4} x\left(\varphi_{1}^{\prime}+\rho_{1}\right)(\bar{\eta}+\bar{c})(\eta+c)\right\}
\end{aligned}
$$

We shall now establish the WT-identities for this action and discuss the $\xi$ independence of the physical observables in it. In order to do this, we shall consider the generating functional

$\mathrm{W}[$ sources $]=\int[\mathrm{D} \varphi] \exp \left\{\mathrm{i} \widehat{S_{\xi}}+[\right.$ source terms $\left.]\right\}$

where

$$
[\mathrm{D} \varphi]=\left[\mathrm{D} \varphi_{1}^{\prime}\right]\left[\mathrm{D} \varphi_{2}^{\prime}\right][\mathrm{D} \psi][\mathrm{D} \bar{\psi}][\mathrm{D} \eta][\mathrm{D} \bar{\eta}]\left[\mathrm{D} A_{\mu}\right]\left[\mathrm{D} B_{\mu}\right]
$$

and the source terms are

[source terms] $=\mathrm{i} \int \mathrm{d}^{4} x\left[\mathrm{~J}^{\mu} \mathrm{B}_{\mu}+\mathrm{K}^{\mu} \mathrm{A}_{\mu}+\chi^{1} \varphi_{1}^{\prime}+\chi^{2} \varphi_{2}^{\prime}+\bar{\lambda} \psi+\bar{\psi} \lambda+\bar{\theta} \eta+\bar{\eta} \theta\right]$

We then have for the gauge variation of $\mathrm{W}$ with respect to the bare gauge parameter $\xi$ keeping other bare parameters fixed

$-\mathrm{i} \frac{\partial W}{\partial \xi}=\int[\mathrm{D} \varphi] \exp \left\{\mathrm{i} \widehat{S_{\xi}}+[\right.$ source terms $\left.]\right\} \frac{\partial \Delta \widehat{S_{\xi}}}{\partial \xi}$

$=\int[\mathrm{D} \varphi] \exp \left\{\mathrm{i} \widehat{S_{\xi}}+[\right.$ source terms $\left.]\right\}\left\{\frac{1}{2 \xi^{2}} \int \mathrm{d}^{4} x\left\{\left(\partial . \widehat{B}^{2}-\xi^{2} \mathrm{M}^{2}{\widehat{\varphi_{2}^{\prime}}}^{2}\right)-2 \mathrm{Mgv} \int \mathrm{d}^{4} x \widehat{\bar{\eta}} \hat{\eta}\right.\right.$ $\left.-2 g \mathrm{M} \int \mathrm{d}^{4} x\left(\varphi_{1}^{\prime}+\rho_{1}\right)(\bar{\eta}+\bar{c})(\eta+c)\right\}$

We shall confine ourselves to the gauge variation around $\xi=1$ :

\footnotetext{
${ }^{4}$ We shall account for the variation of these bare parameters with $\xi$ later in sec.6
} 


$$
\begin{aligned}
& \left.\quad \frac{\partial W}{\partial \xi}\right|_{\xi=1}=\mathrm{i} \int[\mathrm{D} \varphi] \exp \left\{\mathrm{i} \widehat{S_{\xi}}+[\text { source terms }]\right\}\left\{\frac{1}{2} \int \mathrm{d}^{4} x\left\{(\partial . \widehat{B})^{2}-\mathrm{M}^{2}{\widehat{\varphi_{2}^{\prime}}}^{2}\right)-2 \mathrm{Mgv} \int \mathrm{d}^{4} x \widehat{\bar{\eta}} \widehat{\eta}\right. \\
& \left.-2 \mathrm{gM} \int \mathrm{d}^{4} x\left(\varphi_{1}^{\prime}+\rho_{1}\right)(\bar{\eta}+\bar{c})(\eta+c)\right\} \quad(5.9) \\
& =1 / 2 \int \mathrm{d}^{4} x \partial_{\mu} \varepsilon_{B}^{-1} \frac{\delta}{\delta J_{\mu}(x)} \int[\mathrm{D} \varphi] \exp \left\{\mathrm{i} \widehat{S_{F}}+[\text { source terms }]\right\} \varepsilon_{B}^{-1} \partial . B(\mathrm{x}) \\
& -\frac{M^{2}}{2} \int \mathrm{d}^{4} x \varepsilon_{2}^{-1} \frac{\delta}{\delta \chi_{2}(x)} \int[\mathrm{D} \varphi] \exp \left\{\mathrm{i} \widehat{S_{F}}+[\text { source terms }]\right\} \varepsilon_{2}^{-1} \varphi_{2}^{\prime}(\mathrm{x}) \\
& -2 \mathrm{iMgv} \int[\mathrm{D} \varphi] \exp \left\{\mathrm{i} \widehat{S_{\xi}}+[\text { source terms }]\right\} \int \mathrm{d}^{4} x\left\{\hat{\eta} \hat{\eta}+\frac{1}{v}\left(\varphi_{1}^{\prime}+\rho_{1}\right)(\bar{\eta}+\bar{c})(\eta+c\right. \\
& \quad(5.9 \mathrm{a})
\end{aligned}
$$

To simplify this further we use the WT identity at $\xi=1$ :

$0=\int[\mathrm{D} \varphi] \exp \left\{\mathrm{i} \widehat{S_{\xi}}+[\right.$ source terms $\left.]\right\}\left\{\int \mathrm{d}^{4} x\left[\mathrm{~J}^{\mu} \widehat{\delta} \mathrm{B}_{\mu}+\mathrm{K}^{\mu} \widehat{\delta} \mathrm{A}_{\mu}+\bar{\lambda} \widehat{\delta} \psi+\widehat{\delta} \bar{\psi} \lambda+\chi^{1} \widehat{\delta} \varphi_{1}^{\prime}+\right.\right.$ $\left.\chi^{2} \widehat{\delta} \varphi_{2}^{\prime}+\bar{\theta} \widehat{\delta} \eta+\widehat{\delta} \bar{\eta} \theta\right]\left.\right|_{\xi=1}$

To simplify the algebra, we shall set $\bar{\lambda}=\lambda=0$; as these terms do not play a nontrivial part in the algebraic manipulations below.At the end, we shall put back the net contribution of these terms. This gives

$$
\begin{gathered}
0=<<\int \mathrm{d}^{4} x\left[-\mathrm{J}^{\mu} \partial_{\mu} \eta+2 g \chi^{1} \varepsilon_{\varphi_{1}^{\prime}}^{2}\left[\left(\varphi_{2}{ }^{\prime}+\rho_{2}\right)(\eta+c)\right]-2 \mathrm{~g} \chi^{2}\left\{\varepsilon _ { \varphi _ { 2 } ^ { \prime } } ^ { 2 } \left[\left(\varphi_{1}{ }^{\prime}+\rho_{1}\right)(\eta+c\right.\right.\right. \\
)]+v \eta\}+\theta\left[\partial . \mathrm{B}+\mathrm{M} \varphi_{2}{ }^{\prime}\right]>>
\end{gathered}
$$

where $<<\mathrm{O}>>$ stands for $\int[\mathrm{D} \varphi] \mathrm{O} \exp \left\{\mathrm{i} \widehat{S_{\xi}}+[\right.$ source terms $\left.]\right\}$.Differentiating with respect to $\theta(\mathrm{y})$ and setting $\theta=0$, we obtain the WT identity useful for our purpose:

$$
\begin{aligned}
& 0=<<-\mathrm{i}\left[\partial . \mathrm{B}+\mathrm{M} \varphi_{2}{ }^{\prime}\right](\mathrm{y}) \\
& +\int \mathrm{d}^{4} x\left[-\mathrm{J}^{\mu} \partial_{\mu} \eta+2 \mathrm{~g} \chi^{1} \varepsilon_{\varphi_{1}^{\prime}}^{2}\left[\left(\varphi_{2}{ }^{\prime}+\rho_{2}\right)(\eta+c)\right]-2 \mathrm{~g} \chi^{2}\left\{\varepsilon _ { \varphi _ { 2 } ^ { \prime } } ^ { 2 } \left[\left(\varphi_{1}^{\prime}+\rho_{1}\right)(\eta+c\right.\right.\right. \\
& )+\mathrm{v} \eta\}] \overline{\eta(y)}>>\text {. } \\
& 0=<<-\frac{i}{2} \partial . \mathrm{B}(\mathrm{y}) \varepsilon_{B}^{-2}\left[\partial . \mathrm{B}+\mathrm{M} \varphi_{2}{ }^{\prime}\right](\mathrm{y})+\frac{1}{2} \int \mathrm{d}^{4} x\left[-\mathrm{J}^{\mu} \partial_{\mu} \eta+\left[2 \mathrm{~g} \chi^{1} \varepsilon_{\varphi_{1}^{\prime}}^{2}\left(\varphi_{2}{ }^{\prime}+\rho_{2}\right)(\eta+c\right.\right. \\
& )-2 \mathrm{~g} \chi^{2}\left\{\varepsilon_{\varphi_{2}^{\prime}}^{2}\left[\left(\varphi_{1}{ }^{\prime}+\rho_{1}\right)(\eta+c)+\mathrm{v} \eta\right\}\right] \partial . \mathrm{B}(\mathrm{y}) \varepsilon_{\eta}^{-2} \bar{\eta}(\mathrm{y})+\frac{i}{2} \partial^{2} \eta \varepsilon_{\eta}^{-2} \bar{\eta}(\mathrm{y})>>
\end{aligned}
$$

Now operating by $\left[\frac{M i}{2} \frac{\delta}{\delta \chi_{2}} \varepsilon_{\eta}^{-2}\right](\mathrm{y})$ on (5.12), we obtain,

\footnotetext{
${ }^{5}$ As remarked earlier, our model has a chiral anomaly.It can be removed by adding an additional fermion $\Psi$ of appropriate quantum numbers stated in sec. 3 . Then the WT below will contain additional terms that have to be carried forward . They do not however alter the discussion of gauge-independence to one loop qualitatively.
} 


$$
\begin{aligned}
& 0=<<\frac{i M}{2} \varphi_{2}{ }^{\prime} \varepsilon_{B}^{-2}\left[\partial . \mathrm{B}(\mathrm{y})+\mathrm{M} \varphi_{2}{ }^{\prime}(\mathrm{y})\right]+\mathrm{iMg} \varepsilon_{\eta}^{-2} \bar{\eta}(\mathrm{y})\left\{\varepsilon_{\varphi_{2}^{\prime}}^{2}\left[\left(\varphi_{1}{ }^{\prime}+\rho_{1}\right)(\eta+c)(\mathrm{y})\right]+\mathrm{v} \eta\right. \\
& )+\frac{M}{2} \varphi_{2}{ }^{\prime} \varepsilon_{\eta}^{-2} \bar{\eta}(\mathrm{y}) \int \mathrm{d}^{4} x\left[-\mathrm{J}^{\mu} \partial_{\mu} \eta+2 \mathrm{~g} \chi^{1} \varepsilon_{\varphi_{1}^{\prime}}^{2}\left(\varphi_{2}{ }^{\prime}+\rho_{2}\right)(\eta+c)-2 g \chi^{2}\left\{\varepsilon _ { \varphi _ { 2 } ^ { \prime } } ^ { 2 } \left[\left(\varphi_{1}{ }^{\prime}+\rho_{1}\right)(\eta+c\right.\right.\right. \\
& )]+\mathrm{v} \eta\}]>>. \quad(5.14)
\end{aligned}
$$

We now add (5.13) and (5.14) and integrate over $\mathrm{d}^{4} \mathrm{y}$ to obtainf,

$$
\begin{aligned}
& 0=<<\left\{-\frac{i}{2} \int \mathrm{d}^{4} \mathrm{y}\left\{(\partial . \widehat{B})^{2}-\mathrm{M}^{2}{\widehat{\varphi_{2}^{\prime}}}^{2}\right)(\mathrm{y})-\frac{i}{2} \hat{\bar{\eta}} \partial^{2} \widehat{\eta}+\frac{i}{2} \mathrm{M}^{2} \widehat{\bar{\eta}} \widehat{\eta}\right. \\
& \quad+\operatorname{igM} \varepsilon_{\eta}^{-2} \bar{\eta}(\mathrm{y}) \varepsilon_{\varphi_{2}^{\prime}}^{2}\left[\left(\varphi_{1}^{\prime}+\rho_{1}\right)(\eta+c)\right]-\frac{1}{2} \varepsilon_{\eta}^{-2} \bar{\eta}(\mathrm{y})\left[\partial . \mathrm{B}(\mathrm{y})-\mathrm{M} \varphi_{2}{ }^{\prime}\right](\mathrm{y}) \int \mathrm{d}^{4} x\left[-\mathrm{J}^{\mu} \partial_{\mu} \eta+\right. \\
& 2 \mathrm{~g} \chi^{1} \varepsilon_{\varphi_{1}^{\prime}}^{2}\left(\varphi_{2}^{\prime}+\rho_{2}\right)(\eta+c)
\end{aligned}
$$$$
\left.\left.-2 \mathrm{~g} \chi^{2}\left\{\varepsilon_{\varphi_{2}^{\prime}}^{2}\left[\left(\varphi_{1}^{\prime}+\rho_{1}\right)(\eta+c)\right]+\mathrm{v} \eta\right\}\right]\right\}>>\text {. }
$$

We substitute (5.15) in (5.9) to obtain,

$$
\left.\frac{\partial W}{\partial \xi}\right|_{\xi=1}=<<\left\{-\frac{1}{2} \int \mathrm{d}^{4} \mathrm{y} \varepsilon_{\eta}^{-2} \bar{\eta}(\mathrm{y})\left[\partial . \mathrm{B}(\mathrm{y})-\mathrm{M} \varphi_{2}{ }^{\prime}\right](\mathrm{y}) \int \mathrm{d}^{4} x\left[-\mathrm{J}^{\mu} \partial_{\mu} \eta+2 \mathrm{~g} \chi^{1} \varepsilon_{\varphi_{1}^{\prime}}^{2}\left(\varphi_{2}{ }^{\prime}+\rho_{2}\right)(\eta+\right.\right.
$$

c) $\left.)-2 \mathrm{~g} \chi^{2}\left\{\varepsilon_{\varphi_{2}^{\prime}}^{2}\left[\left(\varphi_{1}^{\prime}+\rho_{1}\right)(\eta+c)\right]+\mathrm{v} \eta\right\}\right]+$ ghost terms $>>$

where the net ghost terms are

$$
\begin{aligned}
& \int \mathrm{d}^{4} \mathrm{y}\left\{-\frac{i}{2} \hat{\bar{\eta}} \partial^{2} \hat{\eta}+\frac{i}{2} \mathrm{M}^{2} \hat{\bar{\eta}} \hat{\eta}+\mathrm{igM} \varepsilon_{\eta}^{-2} \bar{\eta}(\mathrm{y}) \varepsilon_{\varphi_{2}^{\prime}}^{2}\left[\left(\varphi_{1}{ }^{\prime}+\rho_{1}\right)(\eta+c)\right]\right. \\
& \left.-2 \operatorname{igM}\left[\mathrm{v} \hat{\bar{\eta}} \hat{\eta}+\left(\varphi_{1}{ }^{\prime}+\rho_{1}\right)(\bar{\eta}+\bar{c})(\eta+c)(\mathrm{y})\right]\right\}
\end{aligned}
$$

These can be simplified using integration by parts in the third term $\left[\varepsilon_{\eta}^{2}=\varepsilon_{\varphi_{2}^{\prime}}^{2}\right]$.We get[,

$$
\frac{i}{2} \bar{\eta} \frac{\delta \widehat{S_{F}}}{\delta \bar{\eta}}-\operatorname{igM} \bar{c}\left(\varphi_{1}^{\prime}+\rho_{1}\right)(\eta+c)
$$

We shall now put back the fermion source terms that we had dropped earlier in the intermediate algebra for our convenience. We thus arrive at the total and simplified expression for $\left.\frac{\partial W}{\partial \xi}\right|_{\xi=1}$ :

$$
\left.\frac{\partial W}{\partial \xi}\right|_{\xi=1}=<<\left\{-\frac{1}{2} \int \mathrm{d}^{4} \mathrm{y} \varepsilon_{\eta}^{-2} \bar{\eta}(\mathrm{y})\left[\partial . \mathrm{B}(\mathrm{y})-\mathrm{M} \varphi_{2}{ }^{\prime}\right](\mathrm{y})\right.
$$

- $\int \mathrm{d}^{4} x\left[-\mathrm{J}^{\mu} \partial_{\mu} \eta+2 \mathrm{~g} \chi^{1} \varepsilon_{\varphi_{1}^{\prime}}^{2}\left(\varphi_{2}{ }^{\prime}+\rho_{2}\right)(\eta+c)-2 \mathrm{~g} \chi^{2}\left\{\varepsilon_{\varphi_{2}^{\prime}}^{2}\left[\left(\varphi_{1}{ }^{\prime}+\rho_{1}\right)(\eta+c)\right]+\mathrm{v} \eta\right\}+\right.$ $\left.\operatorname{ig} \bar{\lambda} \varepsilon_{\psi}^{2} \gamma_{5}(\psi+\zeta)(\eta+c)+\operatorname{ig} \varepsilon_{\psi}^{2}(\bar{\psi}+\bar{\zeta})(\eta+c) \gamma_{5} \lambda\right]+\frac{i}{2} \bar{\eta} \frac{\delta \widehat{S_{F}}}{\delta \bar{\eta}}-\operatorname{igM} \bar{c}\left(\varphi_{1}{ }^{\prime}+\rho_{1}\right)(\eta+c$ )$>>$

We shall utilize the above equation while discussing the $\xi$-independence of

\footnotetext{
${ }^{6}$ We recall $\int \mathrm{a} \varepsilon^{-2} \mathrm{~b}=\int \mathrm{b} \varepsilon^{-2} \mathrm{a}=\int \varepsilon^{-1} \mathrm{~b} \varepsilon^{-1} \mathrm{a}$ etc as easily seen in momentum representation.

${ }^{7}$ We recall that while differentiating the net action,we need not differentiate shadow fields in it as such terms vanish .

${ }^{8}$ Recall the footnote earlier about the chiral anomaly.
} 
the S-matrix.

\section{GAUGE INDEPENDENCE OF THE S-MATRIX ELEMENTS}

In section 5 ,we have presented a modified way of constructing nonlocal field theory for an arbitrary $\xi$, for the spontaneously broken model, by analogy with our work in I. We expect from our results in I, that unlike the usual scheme of Ref.6, the S-matrix elements with this modified regularization are now $\xi$ independent.In the last section, we formulated the WT identity for the model and obtained an expression for $\left.\frac{\partial W}{\partial \xi}\right|_{\xi=1}$ in a simplified form. We shall now use it to verify the gauge-independence in a limited sense: in one-loop approximation and around $\xi=19$. We shall find it necessary to construct a way to deal with the extra ghost term in (5.19), that, as we shall see, is specific to the nonlocal theories. We shall do this in one-loop approximation.

To this end, we shall first make a number of observations:

(a) We have evaluated $\frac{\partial W}{\partial \xi}$ keeping the bare quantities [bare sources, bare masses and couplings] fixed. To show gauge-independence, we need to first evaluate $\left.\frac{\partial W}{\partial \xi}\right|_{R}$ i.e. the derivative with renormalized parameters and sources held fixed. Denoting by $\mathrm{S}^{R}$ and $\mathrm{p}^{R}$ the renormalized sources and parameters [masses and couplings], and by $\mathrm{S}^{U R}$ and $\mathrm{p}^{U R}$, the unrenormalized ones, we can write:

$\left.\frac{\partial W}{\partial \xi}\right|_{R}=\left.\frac{\partial W}{\partial \xi}\right|_{U R}+\left.\sum \frac{\partial S^{U R}}{\partial \xi}\right|_{R} \frac{\delta W}{\delta S^{U R}}+\left.\sum \frac{\partial p^{U R}}{\partial \xi}\right|_{R} \frac{\delta W}{\delta p^{U R}}$

To show gauge independence, we have to show that the contribution to an S-matrix element from $\left.\frac{\partial W}{\partial \xi}\right|_{U R}$ is canceled by the last two terms. We verify this at $\xi=1$. For this purpose, we make several observations on (5.19).

(i) We can ignore the $\partial . J$ term as it does not contribute to Green's functions with physical polarization vectors $[\epsilon \cdot \mathrm{p}=0]$ attached.

\footnotetext{
${ }^{9}$ We recall that the possible gauge-dependent part of S-matrix elements is intrinsically at least one loop; so we can regard within it $\xi_{R}=\xi_{U R}=1$ to this order. So we need not distinguish between the two.
} 
(ii) We can set $\chi_{2}=0$, as it refers to an unphysical scalar.We can also set sources for the ghost fields to zero for similar reasons.

(iii) We can set $\left\langle\left\langle\bar{\eta} \frac{\delta \widehat{S_{F}}}{\delta \bar{\eta}}>>\right.\right.$ to zero by the ghost equation of motion. There, we need to recall that the one-loop measure is independent of $\bar{\eta}$ and the Jacobian term is a constant independent of sources and does not contribute to Green's functions [See e.g. appendix of Ref. 9].

(iv) The source terms proportional to $\chi_{1}, \lambda$ and $\bar{\lambda}$ are of the same kind as they appear in the discussion of the gauge-independence of the local theory [12]; except that they have a modified appearance on account of non-locality. The treatment of these terms is very similar to that for the local theory. To sum up these terms contribute to (a) the two-point functions of the respective fields and this contribution is related to the $\xi$-dependence of the 2-point function of the wave-function of the renormalization;(b) to the on-shell physical Green's functions in the form of external line insertions only ( barring "exceptional momenta" [13]) (c) the net contribution to an S-matrix element is canceled by the second term on the right hand side of (6.1) which has the $\xi$-dependence of Z's.

That leaves us with a term of the term:

- $2 \operatorname{igM} \int \mathrm{d}^{4} \mathrm{y} \bar{c}\left(\varphi_{1}{ }^{\prime}+\rho_{1}\right)(\eta+c)$

We note that if the local limit $(\Lambda \rightarrow \infty)$ is taken first, this term would vanish [ see (6.4) below]; and thus is a new type of term that arises only in the nonlocal model.We shall show that this term does contribute to the 2-point function of the scalar $\varphi_{1}^{\prime}$, and corresponds to a (finite) $\xi$-dependence of the bare mass. In higher point on-shell physical Green's function, it contributes again as ghost tadpole insertions and is similarly canceled by opposite contributions from the last term in (6.1).

By power counting,the above term is an operator of dimension 3. As we shall 
see, moreover, it can be cast as operator of an effective dimension 2. To see this, we recall:

$$
\bar{c}=\left[\frac{\varepsilon_{\eta}^{2}-1}{\Im_{\eta}}\right] \frac{\delta^{P} I[\phi+\psi]}{\delta \eta} \mid
$$

where $\delta^{P}$ denotes partial derivative with respect to a " $\phi$ " keeping " $\psi$ " fixed; and the vertical line implies that the result is to be evaluated at $\psi=\psi[\phi]$. We can simplify this as

$$
\bar{c}=2 \operatorname{Mg}\left[\frac{\varepsilon_{\eta}^{2}-1}{\Im_{\eta}}\right]\left\{\left(\varphi_{1}{ }^{\prime}+\rho_{1}\right)(\bar{\eta}+\bar{c})\right\}
$$

[ as noted earlier, $\bar{c} \rightarrow 0$ as $\Lambda \rightarrow \infty$ ].Thus, this extra term in (6.2) becomes,

$\mathrm{i}(2 \mathrm{Mg})^{2} \int \mathrm{d}^{4} \mathrm{y}\left[\frac{\varepsilon_{\eta}^{2}-1}{\Im_{\eta}}\right]\left\{\left(\varphi_{1}^{\prime}+\rho_{1}\right)(\bar{\eta}+\bar{c})\right\}\left(\varphi_{1}^{\prime}+\rho_{1}\right)(\eta+c)$

where we note two powers of $\mathrm{M}$ before the [nonlocal] operator.

We note several things about the operator in (6.5).

(i) We need to consider the Green's functions only with physical external lines. Then the ghost fields above must necessarily be contracted (in a loop). Then the factor $\left[\frac{\varepsilon_{\eta}^{2}-1}{\Im_{\eta}}\right]$, in momentum space, involves an internal momentum, and thus counts for $(-2)$ momentum dimensions in such diagrams. Thus, it behaves, for power counting purposes, an operator of dimension two.

(ii) The only two-point function of physical fields, to which it can contribute in one loop is the two-point function of $\varphi_{1}^{\prime}$. The corresponding diagram, is by naive power counting, log-divergent. However, in view of the fact that $\left[\frac{\varepsilon_{\eta}^{2}(p)-1}{\Im_{\eta}(p)}\right] \rightarrow 0$ as $\Lambda^{2} \rightarrow \infty$, it is rendered convergent, as a direct calculation also confirms.It leads to a finite contribution to the $\xi$-dependence of the mass of $\varphi_{1}$ '.

(iii) When we consider the insertion of this operator in higher point Green's functions in one loop approximation,we note that there are two kinds of diagrams: one in which the ghost loop has no additional vertices and the other in which the ghost loop has additional lines emerging out of it. The second class of diagrams contain [1-loop] 1PI subgraphs with more than 2 external lines.These subgraphs are convergent by naive power counting itself. In these diagrams, the 
limit $\Lambda^{2} \rightarrow \infty$ can be taken inside. But then as pointed out the diagram contains a factor $\left[\frac{\varepsilon_{\eta}^{2}(p)-1}{\Im_{\eta}(p)}\right]$ that vanishes as $\Lambda^{2} \rightarrow \infty$. Thus, such proper subgraphs [and therefore such one loop diagrams which have no other divergences] vanish as $\Lambda^{2} \rightarrow \infty$ is taken in them. [This has been explicitly verified for the $3-\varphi_{1}$ ' and the $4-\varphi_{1}^{\prime}$ proper vertices]. Also the diagrams having a ghost tadpole ,a finite quantity, attached to a vertex [rather than a line], which can arise when a shadow field in the operator is expended, also vanish as $\Lambda^{2} \rightarrow \infty$. Therefore, the only diagrams for higher point functions, in which this operator insertion matters are those with a ghost tadpole insertion on any one $\varphi_{1}$ ' line [internal or external]. Such contributions to $\left.\frac{\partial W}{\partial \xi}\right|_{U R}$ are canceled by opposite contributions to $\left.\sum \frac{\partial p^{U R}}{\partial \xi}\right|_{R} \frac{\delta W}{\delta p^{U R}}$ with the parameter ' $p$ ' referring to the bare mass of $\varphi_{1}$ '. We further note that in view of the fact that there are no higher point 1PI graphs surviving as $\Lambda^{2} \rightarrow \infty$, correspondingly, there is no $\xi$-dependence induced by this term in bare couplings and $\left.\sum \frac{\partial p^{U R}}{\partial \xi}\right|_{R} \frac{\delta W}{\delta p^{U R}}$ does not have nonvanishing terms when ' $p$ ' refers to any other parameter.

\section{CONCLUSIONS}

We finally summarize our results. We considered, in this work, the elegant nonlocal formulations of gauge theories of Ref.6 and applied it to a simple abelian model with spontaneously broken symmetry and in $\mathrm{R}_{\xi}$-gauges. We applied the process of constructing the nonlocal theory with a finite $\Lambda$ starting from the $\mathrm{R}_{\xi}$-gauge local Lagrangian. We chose a theory with both the vector and axial couplings, as this mimics the Standard Model and conveys our results in a simpler context. We found by explicit calculation that when the formulation of nonlocalization of Ref.[6] was applied to such a model, it lead to a $\xi$-dependent physical observable, the (g-2) for the fermion. [ A similar result had also been checked [10] in the Standard Model earlier]. We then applied the alternate 
procedure of nonlocalization suggested and tested for the unbroken nonabelian gauge theories [9].We formulated the WT identities for this model. As a test of the modified regularization scheme, we also verified the $\xi$-independence of observable S-matrix elements in one loop order in the vicinity of the Feynman gauge.

\section{References}

[1] See for example, Ta-Pei Cheng and Ling-Fong Li in "Gauge Theories of Fundamental Interactions" (Oxford:Clarendon) 1984

[2] G. 't Hooft and M. Veltman Nucl. Phys. B33, 189 (1972)

[3] J.Moffat Phys. Rev. D41,1177(1990)

[4] E. D. Evans et al, Phys Rev D43, 499 (1991)

[5] G. Kleppe and R. P. Woodard, Ann. Phys. (N.Y.) 221, 106 (1993)

[6] G. Kleppe and R. P. Woodard, Nucl. Phys. B388, 81 (1992)

[7] M. A. Clayton Gauge Invariance in nonlocal regularized QED. (Toronto U.) UTPT-93-14, Jul 1993. [hep-th/9307089]

[8] See e.g.N. J. Cornish Mod.Phys.Letts.A 7,1895 (1992); M.A.Clayton et al Int. Jour. Mod. Phys.A 9, 4549 (1994) and references therein.

[9] Anirban Basu and S.D.Joglekar J.Math.Phys. 41,7206 (2000)

[10] S. D. Joglekar and G. Saini,1996 (Unpublished)

[11] S. D. Joglekar and G. Saini, Z. Phys. C.76, 343-353 (1997)

[12] See e.g. B. W. Lee in "Methods in field theory"Les Houches 1975; Editor R. Balian and J.Zinn-Justin 
[13] For a detailed explanation regarding exceptional momenta and why we can ignore gauge dependence for them ,see e.g. S.D.Joglekar Ann.Phys. 109,210(1977).

[14] S.D.Joglekar hep-th/0003104 J.Phys.A (2001) to appear;and S.D.Joglekar hep-th $/ 0003077$ 


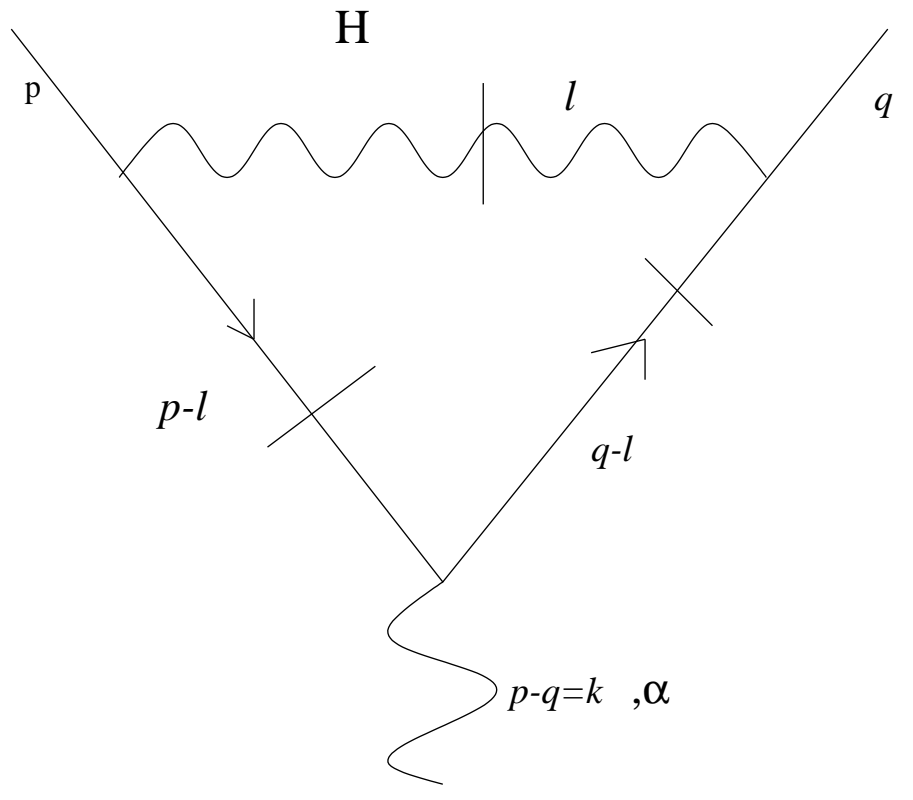

(a)

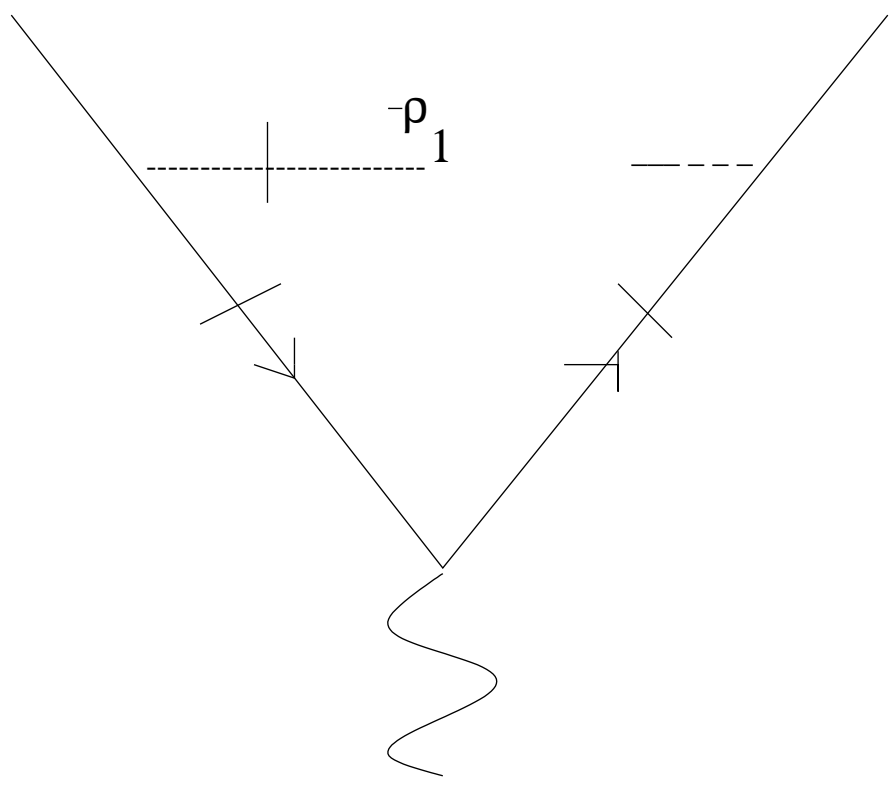

(c)

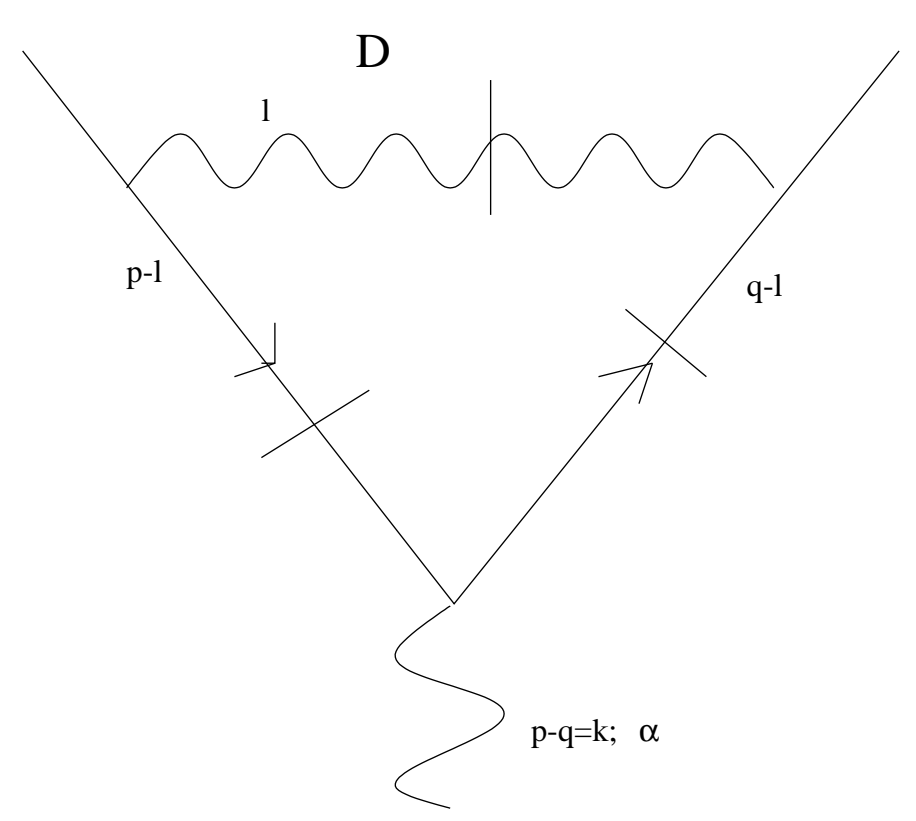

(b)

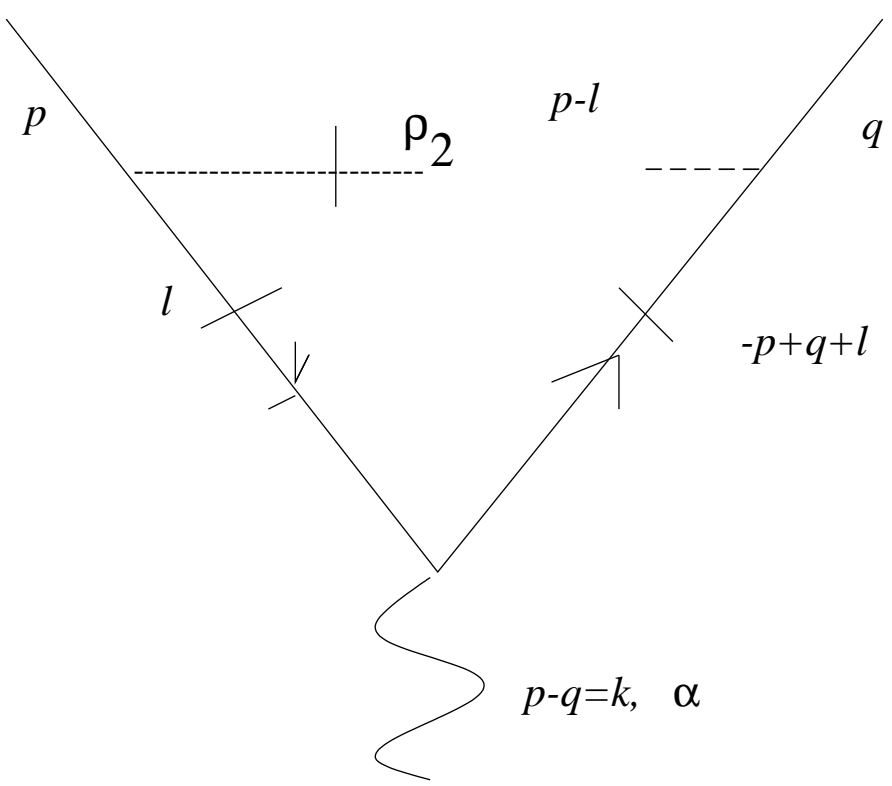

(d)

FIGURE 1: Shadow loop diagrams for (g-2) 